Bio - grafía. Escritos sobre la Biología y su Enseñanza. ISSN 2027-1034

Edición Extraordinaria. p.p. 1501 - 1509

Memorias del IX Encuentro Nacional de Experiencias en Enseñanza de la Biología y la Educación Ambiental. IV Congreso Nacional de Investigación en Enseñanza de la Biología.

\title{
TALLER: "Mapeo del Conocimiento Didáctico del Contenido (CDC) de la alimentación y la nutrición humana"
}

\author{
Dueñas Romero Ana María ${ }^{1}$ \\ Valbuena Ussa Édgar Orlay ${ }^{2}$
}

\section{RESUMEN}

El CDC es el conocimiento que posee el profesor que le permite enseñar un contenido específico a un grupo de estudiantes, con el objetivo de lograr su aprendizaje. En este taller se propone realizar una actividad de mapeo del Conocimiento Didáctico del Contenido de una profesora experimentada cuando enseña la alimentación y la nutrición humana. Para lo cual se analizará el video y la transcripción de un fragmento de clase de la profesora mientras enseña este contenido. Los resultados que se obtengan son de mucha importancia ya que servirán de ejemplo de la forma en que se puede realizar un mapeo del CDC, estableciendo las relaciones que efectúan los diferentes componentes de este conocimiento (contenido, evaluación, estrategias de enseñanza, finalidades de enseñanza, conocimiento sobre los estudiantes, contexto), determinando su frecuencia y analizando el tipo de relaciones que se establecen entre los componentes. Además, permitirá vislumbrar acciones para fortalecer las relaciones de los componentes que lo requieran, bien sea mediante propuestas de desarrollo profesional o ejercicios de reflexión docente.

PALABRAS CLAVE: CDC, mapeo, relaciones, componentes deI CDC.

\section{SUMMARY}

The PCK is the knowledge that the teacher has that allows him to teach a specific content to a group of students, with the aim of achieving their learning. In this workshop it is proposed to carry out an activity of mapping the Pedagogical content knowledge of an experienced teacher when teaching the food and human nutrition. For that the transcription and the video of a fragment of the class of the teacher will be analyzed while it teaches this content. The results

\footnotetext{
${ }^{1}$ Estudiante del DIE Universidad Pedagógica Nacional.E-mail: doc amduenasr879@pedagogica.edu.co. Grupo de investigación Conocimiento Profesional del Profesor de Ciencias

${ }^{2}$ Profesor Universidad Pedagógica Nacional.E-mail: valbuena@pedaqogica.edu.co. Grupo de investigación Conocimiento Profesional del Profesor de Ciencias
} 
Bio - grafía. Escritos sobre la Biología y su Enseñanza. ISSN 2027-1034

Edición Extraordinaria. p.p. 1501 - 1509

Memorias del IX Encuentro Nacional de Experiencias en Enseñanza de la Biología y la Educación Ambiental. IV Congreso Nacional de Investigación en Enseñanza de la Biología.

obtained are of great importance as they will serve as an example of how a mapping of the PCK can be carried out, establishing the relationships that make the different components that compose this knowledge (content, evaluation, teaching strategies, teaching purposes, Students, etc.), establishing their frequency and analyzing the type of relationships that are established between the components. In addition, it will allow to glimpse actions to strengthen the relations of the components that require it, either through proposals of professional development or exercises of teacher reflection

KEY WORDS: PCK, mapping, relationships, PCK components.

\section{INTRODUCCIÓN}

En el mundo los problemas generados por la malnutrición (desnutrición, obesidad y sobrepeso) y las enfermedades asociadas (hipertensión, diabetes, problemas articulares, etc.) están en constante aumento, debido en parte a que los patrones alimentarios están cambiando y en ciertos casos la comida tradicional está siendo remplazada por la denominada "comida chatarra" 3

Para tratar de paliar los efectos de la malnutrición, es necesario brindar una adecuada educación alimenticia y nutricional, la cual jugará un papel muy importante ya que permitirá que los individuos de forma crítica y reflexiva puedan suplir estas necesidades, siendo conscientes de su importancia en el desarrollo de una adecuada salud la cual repercutirá en su calidad de vida.

Teniendo en cuenta lo anterior, se observa la necesidad de efectuar investigaciones que fortalezcan la enseñanza de este contenido, para lo cual se presenta el presente taller, que pretende aportar al estudio del Conocimiento Didáctico del Contenido de la alimentación y la nutrición humana.

EI PCK "es el conocimiento que los profesores desarrollan a través del tiempo y la experiencia, acerca de cómo enseñar un tema particular, para que exista una

\footnotetext{
${ }^{3}$ La comida chatarra es un término acuñado por Michael Jacobson, director del Center for Science in the Public Interest, en 1972, describe la comida que es percibida como insalubre o con poco valor nutritivo.

${ }^{4}$ En el ámbito anglosajón denominado PCK Pedagogical Content Knowledge, término que será utilizado en los estudios cuyos autores lo referencien de esta manera. Sin embargo, los autores de este escrito utilizan el término Conocimiento Didáctico de Contenido debido a que la didáctica hace referencia a la enseñanza de un saber específico y corresponde a la natural eza del CDC.
} 
Bio - grafía. Escritos sobre la Biología y su Enseñanza. ISSN 2027-1034

Edición Extraordinaria. p.p. 1501 - 1509

Memorias del IX Encuentro Nacional de Experiencias en Enseñanza de la Biología y la

Educación Ambiental. IV Congreso Nacional de Investigación en Enseñanza de la Biología.

mayor comprensión por parte del estudiante" (Loughran, Berry \& Mulhall, 2012, p. 9). Fue propuesto por Shulman en 1986, como parte del conocimiento base que deben poseer los profesores para la enseñanza, desde ese entonces las investigaciones para tratar de dilucidar este conocimiento se han desarrollado y en los últimos años son incentivadas debido a su importancia, tal como lo afirma Sandra Abell este conocimiento no es solamente valioso para el desarrollo de investigación educativa, sino que se convierte en un predictor útil para los formadores de profesores de ciencias, es así como actualmente está incluido en los Estándares de Desarrollo Profesional de los Profesores de Ciencias de los Estados Unidos (Garritz y Trinidad-Velasco, 2004) y en Colombia es considerado como uno de los diez pilares para el desarrollo profesional docente (Duque, Celis, Díaz y Gómez, 2014).

EI CDC está conformado por diferentes conocimientos que se relacionan e integran para poder enseñar un contenido espec f́fico. Diferentes estudios y grupos de investigación han propuesto varios tipos de conocimiento que se integran formando el CDC, frente a los cuales no hay consenso. Dueñas, Valbuena, Ravanal \& Rincón (2016), estudiando el Conocimiento Didáctico de Contenido de la alimentación y la nutrición humana a partir de las respuestas dadas al cuestionario semiestructurado ReCo por una profesora que enseña en noveno grado, determinaron como componentes del CDC: el conocimiento sobre el contenido $(\mathrm{C})$, conocimiento sobre las finalidades de enseñanza $(\mathrm{Fl})$, conocimiento sobre las estrategias de enseñanza (ES), conocimiento sobre la evaluación (EV) y el conocimiento del contexto (CTX).

Si bien las investigaciones relacionadas con el CDC han permitido establecer sus componentes, caracterizar cada uno de ellos, establecer como un componente afecta a otros y como un componente particular se relaciona con el conjunto del PCK y con la práctica; Friedrichsen et al. (2010); Aydin y Boz (2013) indican que son escasos los estudios relacionados con la integración entre los componentes, y la naturaleza de éstas (que explican cómo estas integraciones son diferentes en cuanto a su calidad o fuerza). Al respecto se han desarrollado algunos estudios que intentan dilucidar las relaciones que se establecen entre los componentes del CDC, entre ellas: 
Bio - grafía. Escritos sobre la Biología y su Enseñanza. ISSN 2027-1034

Edición Extraordinaria. p.p. 1501 - 1509

Memorias del IX Encuentro Nacional de Experiencias en Enseñanza de la Biología y la

Educación Ambiental. IV Congreso Nacional de Investigación en Enseñanza de la Biología.

Park y Oliver (2008) propone un modelo del CDC a manera de hexágono compuesto por los siguientes componentes: las orientaciones de enseñanza, el conocimiento del curriculum, conocimiento sobre las estrategias de enseñanza, conocimiento sobre la evaluación, conocimiento del entendimiento de los estudiantes y la eficacia del profesor. Estos autores señalan que los componentes se influyen mutuamente en forma continua, indicando que para que la enseñanza sea eficaz los profesores deben integrarlos durante la enseñanza de un contenido específico.

Kaya (2009), estudia las relaciones que se establecen entre los componentes del CDC cuando se enseña el agotamiento de la capa de ozono, mediante el coeficiente de correlación Pearson y el análisis estadístico de la varianza (MANOVA) establece que existen relaciones significativas entre los siguientes componentes del PCK: el conocimiento del curriculum, conocimiento de las dificultades de los estudiantes y el conocimiento de las estrategias-actividades de enseñanza; pero no hay relaciones significativas con el conocimiento de la evaluación, lo cual es explicado porque existen pocos cursos de preparación relacionados con la evaluación en los programas de formación de docentes de ciencias en Turquía, lo que les permite formular sugerencias al respecto. Si bien, este estudio es importante porque establece las relaciones entre componentes, éstas no son modelizadas.

Padilla y Van Driel (2011) estudiando el PCK de la química cuántica en seis profesores, establecieron las relaciones entre los componentes: orientaciones de enseñanza, conocimiento del currículo, de evaluación, de las estrategias de enseñanza, del conocimiento de los estudiantes. Mediante la metodología de PRINCALS, de cada profesor se graficó las relaciones establecidas entre los componentes, encontrando correlaciones entre la mayoría de ellos a excepción del conocimiento de la evaluación, lo cual es explicado en parte porque los profesores consideran que es difícil evaluar la comprensión de conceptos cuánticos en química. Esta investigación reviste una gran importancia porque aporta al conocimiento relacionado con el cómo se pueden establecer las relaciones entre los componentes, graficándolas, indicando su importancia en la caracterización del PCK de los profesores y aportando soluciones al fortalecimiento del Conocimiento Didáctico del Contenido.

Park y Chen (2012) investigan el PCK de cuatro profesores cuando enseñan la fotosíntesis y la herencia. Utilizaron una metodología cualitativa, efectuando el 
Bio - grafía. Escritos sobre la Biología y su Enseñanza. ISSN 2027-1034

Edición Extraordinaria. p.p. 1501 - 1509

Memorias del IX Encuentro Nacional de Experiencias en Enseñanza de la Biología y la

Educación Ambiental. IV Congreso Nacional de Investigación en Enseñanza de la Biología.

análisis en profundidad, el enfoque enumerativo y el método comparativo constante. Los resultados fueron presentados en un modelo o mapa en forma de pentágono, compuesto por cinco componentes del PCK: orientaciones para la enseñanza, conocimiento del currículo, de la evaluación, de las estrategias de enseñanza y del entendimiento de los estudiantes. Esta investigación tiene una gran importancia porque proponen un mapa en el cual se pueden se evidencia las relaciones que establecen los componentes del PCK y su frecuencia, de forma tal que se puede analizar sus interacciones, para tratar de explicarlas y proponer acciones para mejorar la calidad del PCK de los profesores. Algunos resultados de esta investigación indican que: el PCK es idiosincrático y tópico específico, el conocimiento sobre la comprensión de los estudiantes y las estrategias de enseñanza fueron centrales en la integración, el conocimiento sobre el currículo tuvo las relaciones más limitadas con otros componentes.

Aydin y Boz (2013) examinan la integración de los componentes del PCK de dos profesores cuando enseñan: la electroquímica celular y las reacciones redox. Para lo cual utilizan una metodología cualitativa, mediante el análisis en profundidad, el enfoque enumerativo y el método comparativo constante. Los resultados son presentados en el mapa compuesto por cinco componentes del PCK. Esta investigación aporta al mapeo al darle un valor entre 1 a 3 a cada relación que se establece, teniendo en cuenta la calidad de las relaciones entre los componentes del PCK, evaluadas de acuerdo a las acciones que realiza el profesor para que los estudiantes sean partícipes de su aprendizaje. Entonces en este mapa no solamente se puede observar las relaciones que establecen los componentes del PCK y su frecuencia, sino también las relaciones son representadas según sea el caso por líneas de diferente grosor dependiendo la calidad de éstas. Los resultados obtenidos en esta investigación establecen que: las integraciones fueron idiosincráticas y tópico específicas, algunas integraciones son simples y otras complicadas, algunas son más frecuentes que otras, las orientaciones para la enseñanza son un componente primordial que moldea las decisiones instruccionales de los profesores.

Ravanal y López-Cortes (2016) investigan a nivel declarativo el CDC de la célula en cinco profesores de Biología, analizando los componentes del Conocimiento Didáctico de Contenido propuestos por Magnusson, Krajcik and Borko (1999); Park y Oliver (2008): orientaciones de enseñanza, evaluación, currículo, estrategias de enseñanza y conocimiento del entendimiento de los estudiantes. 
Bio - grafía. Escritos sobre la Biología y su Enseñanza. ISSN 2027-1034

Edición Extraordinaria. p.p. 1501 - 1509

Memorias del IX Encuentro Nacional de Experiencias en Enseñanza de la Biología y la Educación Ambiental. IV Congreso Nacional de Investigación en Enseñanza de la Biología.

Metodológicamente efectúan el análisis de contenido y el enumerativo. Algunos resultados son presentados en el mapa propuesto por Oliver y Chen (2012), esta investigación aporta al modelo la direccionalidad de las relaciones y relaciona el mapeo del CDC con el enfoque didáctico de los profesores, planteando acciones puntuales relacionadas con programas de desarrollo profesional docente.

Los estudios referenciados anteriormente proporcionan valiosa información sobre el mapeo del CDC como herramienta que permite identificar las relaciones que se establecen entre sus componentes e identificar sus frecuencias. Además, la valoración que se da a las relaciones establecidas permite establecer cuáles deberían fortalecer su calidad, en este punto se podrían proponer estrategias para fortalecer el CDC de los profesores o los programas de formación docente. De ahí la importancia de proponer este taller en el cual se darán ciertas bases para mapear el CDC de una profesora cuando enseña la alimentación y la nutrición humana, para lo cual se tendrá en cuenta en la valoración de las relaciones que se establecen una hipótesis de progresión, que ha sido construida por los talleristas, en la cual se da un valor de 1 a las relaciones con niveles de complejidad inicialtradicional, un valor de 2 a niveles de complejidad intermedios, tecnológico o espontaneista y 3 a niveles de complejidad de referencia-constructivista.

\section{PROPÓSITOS}

$\checkmark$ Realizar un ejercicio de caracterización del CDC de la alimentación y la nutrición humana.

$\checkmark$ Reflexionar sobre la práctica de una profesora en la enseñanza de la alimentación y la nutrición humana.

$\checkmark$ Discutir las potencialidades del mapeo del CDC de la alimentación y la nutrición humana, en la enseñanza de dicho contenido

$\checkmark$ Discutir sobre las implicaciones didácticas del mapeo del CDC

\section{ASPECTOS PROCEDIMENTALES}

DURACIÓN: 75 minutos

MATERIALES: 30 hojas de papel blanco tamaño A4, 30 copias de la transcripción de los fragmentos de la clase, 30 lápices, 30 esferos, video beam, computador, micrófono y marcadores. 
Bio - grafía. Escritos sobre la Biología y su Enseñanza. ISSN 2027-1034

Edición Extraordinaria. p.p. 1501 - 1509

Memorias del IX Encuentro Nacional de Experiencias en Enseñanza de la Biología y la

Educación Ambiental. IV Congreso Nacional de Investigación en Enseñanza de la Biología.

\section{ACTIVIDADES}

Momento 1: Introducción al taller, presentación de los objetivos: 5 minutos

Momento 2: Descripción de las actividades: 10 minutos

ACTIVIDAD 1. Los participantes observarán durante 10 minutos un video correspondiente a un fragmento de la clase de una profesora al enseñar contenidos sobre alimentación y la nutrición humana. Después se les pedirá que se organicen en grupos y se dará a cada uno la transcripción del fragmento de video, lo cual servirá de insumo para realizar las actividades y responder a las siguientes situaciones:

a. Mediante un esquema representen la práctica de la profesora, teniendo en cuenta: qué enseña, para qué lo enseña, cómo lo enseña, cómo evalúa, qué conocimiento posee sobre los estudiantes (10 minutos)

b. A partir de la estructuración del esquema se hará la plenaria, donde se analizarán las respuestas de los participantes (10 minutos)

ACTIVIDAD 2. Socialización de la manera en que los talleristas mapearon la práctica de la profesora. (15 minutos), para lo cual se distribuirán entre los participantes, fotocopias con el modelo en forma de pentágono a partir del propuesto por Park y Chen (2012)

ACTIVIDAD 3. Discusiones y conclusiones relacionadas con el mapeo del CDC. (15 minutos)

\section{REFERENCIAS BILIOGRÁFICAS}

Aydin, S., \& Yezdan, B. (2013). The nature of integration among PCK components: A case study of two experienced chemistry teachers. Chemistry Education Research and Practice, 14, 615-624.

Dueñas, A., M., Valbuena, E., Ravanal, E., \& Rincón, M. (2016). Mapeo del conocimiento didáctico del contenido de la alimentación y la nutrición humana de una profesora de secundaria. Revista Tecné, Episteme y Didaxis: TED, Número extraordinario, 238-245.

Duque, M., Celis, J., Diaz, B., \& Gómez, M. . (2014). Diez pilares para un programa de desarrollo profesional docente centrado en el aprendizaje de los estudiantes. Revista Colombiana de Educación, 67, 107-124. 
Bio - grafía. Escritos sobre la Biología y su Enseñanza. ISSN 2027-1034

Edición Extraordinaria. p.p. 1501 - 1509

Memorias del IX Encuentro Nacional de Experiencias en Enseñanza de la Biología y la

Educación Ambiental. IV Congreso Nacional de Investigación en Enseñanza de la Biología.

Garritz, A., \& Trinidad Velasco, R. (2004). El conocimiento pedagógico del contenido. Educación Química, 15(2), 2-6.

Kaya, O. (2009). The Nature of Relationships among the Components of Pedagogical Content Knowledge of Preservice Science Teachers: " Ozone layer depletion" as an example. International Journal of Science Education, 31(7), 961-988. doi: 10.1080/09500690801911326

Lorenzo, M., Garritz, A., \& Daza,S. (2014). ¿Transposición didáctica o conocimiento didáctico del contenido o conocimiento pedagógico del contenido? "A rose by any other name" Un recuerdo de Sandy Abell. In A. Garritz, Lorenzo, M.,\& Daza,S (Ed.), Conocimiento Didáctico del Contenido. Una perspectiva Iberoamericana (pp. 2-12). Saarbrucken, Alemania: Editorial Académica Española.

Loughran, J., Berry, A., \& Mulhall, P. (2012). Understanding and Developing Science Teachers' Pedagogical Content Knowedge (J. Loughran Ed. Second Edition ed.). Rotterdam: Sense Publishers.

Magnusson, S., Krajcik, j., \& Borko,H. (1999). Nature, Sources and Development of Pedagogical Content Knowledge for Science Teaching. In J. L. GessNewsome, N (Ed.), Examining Pedagogical Content Knowedge. United States of America: Kluwer Academic Publishers.

Padilla, K., \& Van Driel, J. . (2011). The relationships between PCK components: the case of quantum chemistry professors. Chemistry Education Research and Practice, 12, 367-378. doi: 10.1039/C1RP90043A

Park, S., \& Chen, Y. (2012). Mapping out the Integration of the Components of Pedagogical Content Knowledge (PCK): Examples From High School Biology Classrooms. Journal of research in science teaching, 49(7), 922941. doi: 10.1002/tea.21022

Park, S., \& Oliver, S. (2008). Revisiting the Conceptualisation of Pedagogical Content Knowledge (PCK): PCK as a Conceptual Tool to Understand Teachers as Profesionals. Research in Science Education, 38(3), 261-284. doi: 10.1007/s11165-007-9049-6 
Bio - grafía. Escritos sobre la Biología y su Enseñanza. ISSN 2027-1034

Edición Extraordinaria. p.p. 1501 - 1509

Memorias del IX Encuentro Nacional de Experiencias en Enseñanza de la Biología y la Educación Ambiental. IV Congreso Nacional de Investigación en Enseñanza de la Biología.

\section{ANEXO 1}

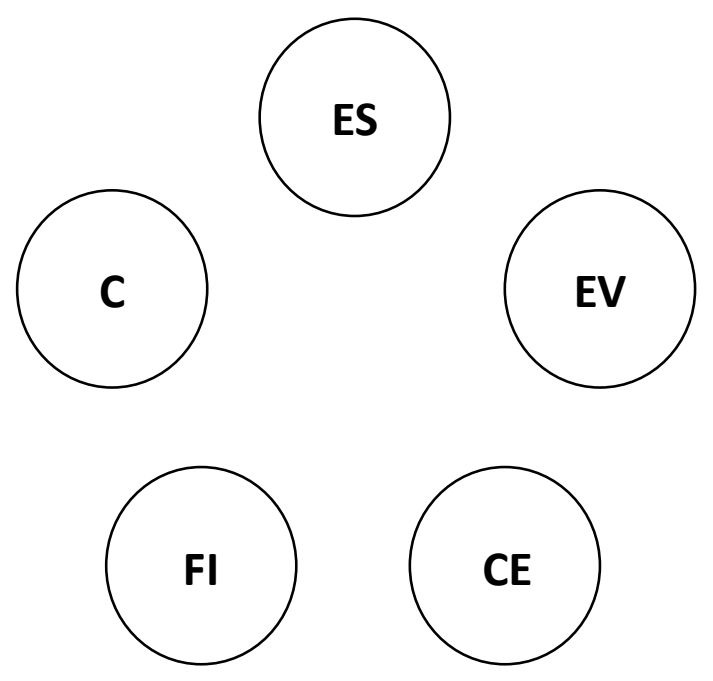

NIVEL DE COMPLEJIDAD

INICIAL [1-1,67]

INTERMEDIO (1,67-2,34]

REFERENCIA $(2,34-3]$

Figura 1. Modelo pentagonal para mapear el CDC modificado a partir del propuesto por Park y Chen (2012) 